\title{
Power analysis of RC6 and SERPENT*
}

\author{
WU WENLING, FENG DENGGUO, QING SIHAN
}

(Engineering Research Center for Information Security Technology,

Chinese Academy of Sciences, Beijing 100080,China)

(Laboratory of Computer Science, Institute of Software, Chinese Academy of Sciences, Beijing 100080,China)

Key words: Key, Algorithm, Power analysis

Abstract: We present power analysis on RC6 and SERPENT which are AES finalist candidates, Our approach shows that the power analysis on RC6 needs $2^{212}$ and $2^{172}$ trials on average for 256 and 192 bit key respectively. The power analysis on SERPENT needs $2^{160} 2^{120}$ and $2^{80}$ trials on average for 256192 and 128 bit key respectively. Although the number of trials is too big to implement, the approach presented in this paper reduces greatly the key size of RC6 and SERPENT. SERPENT have many weak keys for power analysis. Also we give two modified key schedules for SERPENT such that our attack is no longer possible.

\section{INTRODUCTION}

P. Kocher, J.Jaffe, and B.Jun ${ }^{[1]}$ presented power analysis on smart card implementations of the cipher algorithms which is easy to carry out and very effective in practice. The basic idea behind power analysis is that the power consumed by the smart card at any particular time during the cryptographic operation is related to the instruction being executed and to the data being processed. For instance, multiplication consumes more power than addition, and writing 1 's consumes more power than writing 0 's. Biham

* Supported by 973 Project (No.G1999035802) and NSF (No.19931010)

The original version of this chapter was revised: The copyright line was incorrect. This has been corrected. The Erratum to this chapter is available at DOI: 10.1007/978-0-387-35515-3_53 
and Shamir ${ }^{[2]}$ introduced a variant of power analysis, in which the attacker need not to know either the inputs or the outputs of the encryption algorithm, and need not to know the software implementation details in the smart card. For the sake of simplicity, we assume that the protocol used in the smart card always performs its sub-protocols in the same order, and always requires same number of clock cycles for executing each sub-protocol. As a result, we can align the power consumption graphs of different executions of the protocol, then compare consumed power of each instruction.

The attack can be performed in two steps:

Step1, Attacker finds the tiny sections of the power consumption graph which are related to the key scheduling parts of the encryption operations. The attacker can accomplish this task in two sub-steps.

1) The attacker executes a large number of trials on a single smart card for different data, then compares the power consumption graphs at each clock cycle, and discards those clock cycles in which the graphs show a significant variability of the power consumption, duo to differences of the processed data. The remaining clock cycles represent operations which are data independent.

2) The attacker repeats 1) for several smart cards with different keys, and finds their common data independent regions. Among these regions, The attacker discards the clock cycles which have small variability among the different cards. The remaining clock cycles which are related to the key schedule are needed by the attacker.

Step2, The attacker collects the Hamming weights of each byte of sub-keys. In software implementations of iterated block cipher in 8-bit smart cards usually compute each sub-keys just before it is used, since their small RAM capability makes it difficult to calculate all the sub-keys in advance. The sub-keys are computed and stored in RAM in chunks of 8 bits, and consumed power during the write operation is related to the number of 1's among the 8 written bits. So it is possible to get the Hamming weights of each byte of sub-keys.

Step3, The attacker studies the key scheduling algorithm carefully, extracts the master key or information about the master key by using the Hamming weights of each byte of sub-keys.

It has been pointed ${ }^{[2]}$ that step 1 and step 2 are easy to realize practically. Therefore, it is assumed in this paper that the attacker knows the Hamming weights of each byte of sub-keys.

NIST started to collect AES(Advanced Encryption Standard) candidates in 1997, and publicized five finalist candidates in 1999 which include RC6 and SERPENT. It has been shown that the data requirements for successful differential attack or linear attack on RC6 and SERPENT exceed $2^{128}$, the total number of possible plaintexts. The results in this paper show that the power attack on RC6 needs $2^{212}$ and $2^{172}$ trials on average for 256 and 192 bit key respectively. The power attack on SERPENT needs $2^{160} 2^{120}$ and $2^{80}$ 
trials on average for 256192 and 128 bit key respectively. Although the number of trials is too big to realize, the approach presented in this paper reduces greatly the key size of RC6 and SERPENT.

This paper is organised as follows. First we describe the key schedule for RC6. In Sect.3 we give power attack on the key schedule of RC6. Next we describe the key schedule for SERPENT. In Sect.5 we give power analysis on the key schedule of SERPENT. Finally we present our conclusion and two modified key schedules.

\section{KEY SCHEDULE FOR RC6-W/R/B}

Input: User -supplied b byte key preloaded into the c-word array $\mathrm{L}[0, \ldots \mathrm{c}-1]$

Number $r$ of rounds

Output: w-bit round keys $\mathrm{S}[0, \ldots 2 \mathrm{r}+3]$

Procedure: $S[0]=P_{w}$

For $\mathrm{i}=1$ to $2 \mathrm{r}+3$ do

$\mathrm{S}[\mathrm{i}]=\mathrm{S}[\mathrm{i}-1]+\mathrm{Q}_{\mathrm{w}}$

$A=B=i=j=0$

$\mathrm{v}=3 \times \max \{\mathrm{c}, 2 \mathrm{r}+4\}$

for $\mathrm{s}=1$ to $\mathrm{v}$ do

\{

$$
\begin{aligned}
& A=S[i]=(S[i]+A+B)<<<3 \\
& B=L[j]=(L[j]+A+B)<<<(A+B) \\
& i=(i+1) \bmod (2 r+4) \\
& j=(j+1) \operatorname{modc}
\end{aligned}
$$

3. POWER ANALYSIS ON THE KEY SCHEDULE OF RC6

Let $A_{0}=S[0], \ldots, A_{2 r+3}=S[2 r+3]$ be sub-keys, whose Hamming weights are known. $\mathrm{A}$ and $\mathrm{B}$ are inputs of some round whose outputs are $\mathrm{A}_{0}$ and $\mathrm{B}_{0}$, we have the following equations:

$$
\begin{aligned}
& A_{0}=(S[0]+A+B) \ll<3 \\
& B_{0}=\left(L[0]+A_{0}+B\right)<<\left(A_{0}+B\right) \\
& A_{1}=\left(S[1]+A_{0}+B_{0}\right) \ll<3 \\
& B_{1}=\left(L[1]+A_{1}+B_{0}\right) \ll<\left(A_{1}+B_{0}\right)
\end{aligned}
$$




$$
\begin{aligned}
& A_{2}=\left(S[2]+A_{1}+B_{1}\right) \ll<3 \\
& B_{2}=\left(L[2]+A_{2}+B_{1}\right) \ll<\left(A_{2}+B_{1}\right) \\
& A_{3}=\left(S[3]+A_{2}+B_{2}\right) \ll<3 \\
& B_{3}=\left(L[3]+A_{3}+B_{2}\right) \ll<\left(A_{3}+B_{2}\right) \\
& A_{4}=\left(S[4]+A_{3}+B_{3}\right) \ll<3 \\
& B_{4}=\left(L[4]+A_{4}+B_{3}\right) \ll<\left(A_{4}+B_{3}\right) \\
& A_{5}=\left(S[5]+A_{4}+B_{4}\right) \ll<3 \\
& B_{5}=\left(L[5]+A_{5}+B_{4}\right) \ll<\left(A_{5}+B_{4}\right) \\
& A_{6}=\left(S[6]+A_{5}+B_{5}\right) \ll<3 \\
& B_{6}=\left(L[6]+A_{6}+B_{5}\right) \ll<\left(A_{6}+B_{5}\right)
\end{aligned}
$$

Step 1 , For any $(A, B)$, predict $\mathrm{L}[0]$ by using equations(1)and(2).

At first, compute $\mathrm{A}_{0}$ by using equation (1). From equations (1) and (2), we can get the following equation:

$$
A_{1}=\left\{S[1]+A_{0}+\left[\left(L[0]+A_{0}+B\right)<<\left(A_{0}+B\right)\right]\right\}<<<3
$$

Because we know $A_{0}$ and $B$, we can predict L[0] by using the Hamming weights of each byte of $A_{1}$.

The average number of $\mathrm{L}[0]$ is $2^{20}$.

Step 2, For any $L[0]$, compute $B_{0}$ and $A_{1}$; then predict L[1] by using equations (2)and(3) as similar as step 1.

The average number of $L[1]$ is $2^{20}$.

Step 3, for any $(L[0], L[1])$, compute $B_{1}$ and $A_{2}$; then predict L[2] by using equations (3) and (4) as similar as step 1.

The average number of $L[2]$ is $2^{20}$.

Step 4, for any $(L[0], L[1], L[2])$, compute $B_{2}$ and $A_{3}$; then predict L[3] by using equations (4) and (5) as similar as step 1 .

The average number of $L[3]$ is $2^{20}$.

Step 5, for any $(L[0], L[1], L[2], L[3])$, compute $B_{3}$ and $A_{4}$; then predict $\mathrm{L}[4]$ by using equations (5) and (6) as similar as step 1 .

The average number of $L[4]$ is $2^{20}$.

Step 6 , for any $(L[0], L[1], L[2], L[3], L[4])$, compute $B_{4}$ and $A_{5}$; then predict $L[5]$ by using equations (6) and (7) as similar as step 1 .

The average number of $L[5]$ is $2^{20}$. 
Finally, test $2^{120}(L[0], L[1], L[2], L[3], L[4], L[5])$. For any $A$, as the Hamming weights of each byte of $A_{0}$ are known, the average number of $\mathrm{B}$, which make $(A, B)$ satisfy equation (1), is $2^{20}$, So the average number of $(A, B)$ is $2^{52}$. Therefore, the above attack on RC6 needs $2^{172}$ trials for 192 bit key. Similarly, the above attack on RC6 needs $2^{212}$ trials for 256 bit key.

\section{KEY SCHEDULE OF SERPENT}

At first, pad the master key to 256 bits and write it as eight 32-bit words $w_{-8}, \ldots, w_{-1}$.

Next, make 132 32-bit words $w_{0}, \ldots, w_{131}$ by using the following affine recurrence: $\quad w_{i}=\left(w_{i-8} \oplus w_{i-5} \oplus w_{i-3} \oplus w_{i-1} \oplus \phi \oplus i\right)<<<11$

where $\varphi$ is the fractional part of the golden ratio $(\sqrt{5}+1) / 2$ or $0 \mathrm{x} 9 \mathrm{e} 3779 \mathrm{~b} 9$ in hexadecimal.

Finally, construct subkeys by using S-boxes in the following way

$$
\begin{aligned}
K_{0}= & S_{3}\left(w_{0}, w_{1}, w_{2}, w_{3}\right) \\
K_{1}= & S_{2}\left(w_{4}, w_{5}, w_{6}, w_{7}\right) \\
& \ldots \ldots \\
K_{i}= & S_{j}\left(w_{4 i}, w_{4 i+1}, w_{4 i+2}, w_{4 i+3}\right) \quad 3 \equiv(i+j) \bmod 8 \\
& \ldots \ldots . \\
K_{32}= & S_{3}\left(w_{128}, w_{129}, w_{130}, w_{131}\right)
\end{aligned}
$$

\section{POWER ANALYSIS ON THE KEY SCHEDULE OF SERPENT}

Let $X, Y \in G F(2)^{8} \quad S_{j}(X)=Y \quad S_{j}: G F(2)^{8} \rightarrow G F(2)^{8} \quad$ be $\quad$ a permutation. Given $W_{H}(Y)$ the number of $\mathrm{X}$ satisfying $W_{H}\left(S_{j}(X)\right)=W_{H}(Y)$ is denoted as $N(X)$ which is about 32 on average. In the key schedule of SERPENT

$$
\begin{aligned}
& K_{i}=\left(k_{4 i}, k_{4 i+1}, k_{4 i+2}, k_{4 i+3}\right)=S_{j}\left(w_{4 i}, w_{4 i+1}, w_{4 i+2}, w_{4 i+3}\right) \\
& k_{4 i+t}=S_{j}\left(w_{4 i+t}\right) \quad 0 \leq t \leq 3 \\
& k_{4 i+t}=\left(k_{4 i+t}^{1}, k_{4 i+t}^{2}, k_{4 i+t}^{3}, k_{4 i+t}^{4}\right)=S_{j}\left(w_{4 i+t}^{1}, w_{4 i+t}^{2}, w_{4 i+t}^{3}, w_{4 i+t}^{4}\right)
\end{aligned}
$$




$$
\left\{\begin{array}{l}
k_{4 i+t}^{1}=S_{j}\left(w_{4 i+t}^{1}\right) \\
k_{4 i+t}^{2}=S_{j}\left(w_{4 i+t}^{2}\right) \\
k_{4 i+t}^{3}=S_{j}\left(w_{4 i+t}^{3}\right) \\
k_{4 i+t}^{4}=S_{j}\left(w_{4 i+t}^{4}\right)
\end{array}\right.
$$

Let $N\left(w_{4 i+t}^{1}\right)$ denote the number of $w_{4 i+t}^{1}$ which satisfy $W_{H}\left(S_{j}\left(w_{4 i+t}^{1}\right)\right)=W_{H}\left(k_{4 i+t}^{1}\right), N\left(w_{4 i+t}^{2}\right)$ denote the number of $w_{4 i+t}^{2}$ which satisfy $W_{H}\left(S_{j}\left(w_{4 i+t}^{2}\right)\right)=W_{H}\left(k_{4 i+t}^{2}\right), N\left(w_{4 i+t}^{3}\right)$ denote the number of $w_{4 i+t}^{3}$ which satisfy $W_{H}\left(S_{j}\left(w_{4 i+t}^{3}\right)\right)=W_{H}\left(k_{4 i+t}^{3}\right), N\left(w_{4 i+t}^{4}\right)$ denote the number of $w_{4 i+t}^{4}$ which satisfy $W_{H}\left(S_{j}\left(w_{4 i+t}^{4}\right)\right)=W_{H}\left(k_{4 i+t}^{4}\right)$. If $W_{H}\left(k_{4 i+t}^{1}\right) W_{H}\left(k_{4 i+t}^{2}\right) \quad W_{H}\left(k_{4 i+t}^{3}\right)$ and $W_{H}\left(k_{4 i+t}^{4}\right)$ are known, then the number of candidates of $w_{4 i+t}$ is $N\left(w_{4 i+t}\right)=N\left(w_{4 i+t}^{1}\right) \times N\left(w_{4 i+t}^{2}\right) \times N\left(w_{4 i+t}^{3}\right) \times N\left(w_{4 i+t}^{4}\right)$. So there are $N\left(w_{l}\right)$ candidates for each $w_{l}(0 \leq l \leq 131)$. Because $w_{0}, \ldots, w_{131}$ is expanded from the master key $w_{-8}, \ldots, w_{-1}$ by the affine recurrence we only need to know $w_{0}, \ldots, w_{7}$ for computing $w_{8}, w_{9}, \ldots, w_{131}$ and getting $w_{-8}, \ldots, w_{-1}$. We extract $w_{0}, \ldots, w_{7}$ in the following way:

Step1, choose $\left(w_{0}, \ldots, w_{7}\right.$ there are about $N\left(w_{0}\right) \times \ldots \times N\left(w_{7}\right)$.

Step2, compute $w_{l}(8 \leq l \leq 131)$ by the affine recurrence and test whether $W_{H}\left(S_{j}\left(w_{l}\right)\right)$ and each byte Hamming weight known are same;

if no give up this candidate of $\left(w_{0}, \ldots, w_{7}\right)$ return step 1

if yes compute $w_{l+1}$.

Step3, the remaining $\left(w_{0}, \ldots, w_{7}\right.$ should be only one.

The complexity of above attack depends on $N\left(w_{0}\right) \times \ldots \times N\left(w_{7}\right)$. For 32bit word $w_{i}=\left(w_{i}^{1}, w_{i}^{2}, w_{i}^{3}, w_{i}^{4}\right)$ because the average number of $N\left(w_{i}^{1}\right) \quad N\left(w_{i}^{2}\right) \quad N\left(w_{i}^{3}\right)$ and $N\left(w_{i}^{4}\right)$ are all 32 the average number of $N\left(w_{i}\right)$ is $2^{20}$. So above attack on SERPENT needs $2^{160} 2^{120}$ and $2^{80}$ trials on average for 256192 and 128 bit key respectively.

SERPENT have weak key for above attack. We explain this for 128-bit key. Let $K=\left(w_{-8}, w_{-7}, w_{-6}, w_{-5}\right)$ and $w_{-4}=w_{-3}=w_{-2}=w_{-1}=0$. For any $w_{-8}$ and $\quad w_{-7}$ let $\quad w_{-5}=w_{-8} \oplus \phi \quad$ and 
$w_{-6}=\phi \oplus 2 \oplus\left(w_{-7} \oplus \phi \oplus 1\right)<<11$ then

$w_{0}=w_{2}=0 \quad w_{1}=\left(w_{-7} \oplus \phi \oplus 1\right)<<11 \quad w_{3}=\left(w_{-8} \oplus 3\right)<<11$ so

above attack needs $2^{39}$ trials on average. There are $2^{64}$ keys having this property.

If choosing $w_{-8}$ and $w_{-7}$ such that $w_{1}$ and $w_{3}$ satisfy the following equation

$$
\begin{aligned}
& k_{1}=\left(k_{1}^{1}, k_{1}^{2}, k_{1}^{3}, k_{1}^{4}\right)=S_{3}\left(w_{1}\right) \\
& k_{3}=\left(k_{3}^{1}, k_{3}^{2}, k_{3}^{3}, k_{3}^{4}\right)=S_{3}\left(w_{3}\right) \\
& 0 \leq W_{H}\left(k_{t}^{i}\right) \leq 2 \quad 6 \leq W_{H}\left(k_{t}^{i}\right) \leq 8 \quad 1 \leq i \leq 4 \quad t=1,3
\end{aligned}
$$

$\mathrm{W}_{\mathrm{H}}\left(k_{t}^{i}\right)$ have six candidates. When $\mathrm{W}_{\mathrm{H}}\left(k_{t}^{i}\right)=08$ the i-th byte of word $w_{t}$ have two candidates when $\mathrm{W}_{\mathrm{H}}\left(k_{t}^{i}\right)=17$ the $\mathrm{i}$-th byte of word $w_{t}$ have 16 candidates when $k_{t}^{i}=26$ the $i$-th byte of word $w_{t}$ have 56 candidates. Therefore, $w_{1}$ and $w_{3}$ satisfying equation $(8)$ have $(2 \times 37)^{4}$ respectively, and the distribution number of byte-Hamming weight of $k_{1}$ and $k_{3}$ have $6^{4}$ respectively. The average candidates of $w_{1}$ and $w_{3}$ is $\left(\frac{37}{3}\right)^{4}$. So the trial number for key satisfying (8) is less than $2^{29}$ on average, these keys are about $(2 \times 37)^{8}>2^{48}$.

If change the condition (8) as follows:

$$
0 \leq W_{H}\left(k_{t}^{i}\right) \leq 1 \quad 7 \leq W_{H}\left(k_{t}^{i}\right) \leq 8
$$

Then the trial number for key satisfying (9) is $\left(\frac{9}{2}\right)^{4} \times\left(\frac{9}{2}\right)^{4}$ on average, these keys are about $18^{8}>2^{33}$.

\section{CONCLUSIONS}

The five finalist AES candidates have been shown that they are resistant to differential cryptanalysis, linear cryptanalysis and related-key cryptanalysis. In this paper we have proven that if they are used in smart cards, the power analysis on RC6 needs $2^{212}$ and $2^{172}$ trials on average for 256 and 192 bit key respectively. We also show that the power analysis on SERPENT needs $2^{160} 2^{120}$ and $2^{80}$ trials on average for 256192 and 128 bit key respectively. Although the number of trials is too big to implement, the approach 
presented in this paper reduces greatly the key size of RC6 and SERPENT. SERPENT have many weak keys for the above power analysis. We give two modified key schedules for SERPENT such that the above attack cannot be effected.

The first is as follows:

Step 1, pad the master key to 256 bits and write it as eight 32-bit words $w_{-8}, \ldots, w_{-1}$.

Step 2, make 264 32-bit words $w_{0}, \ldots, w_{131}, \ldots, w_{263}$ by using the following affine recurrence:

$$
w_{i}=\left(w_{i-8} \oplus w_{i-5} \oplus w_{i-3} \oplus w_{i-1} \oplus \phi \oplus i\right)<<<11
$$

where $\varphi$ is the fractional part of the golden ratio $(\sqrt{5}+1) / 2$ or $0 \times 9 \mathrm{e} 3779 \mathrm{~b} 9$ in hexadecimal.

Step3, construct $K_{i}^{*}(0 \leq i \leq 65)$ by using S-boxes in the following way

$$
\begin{aligned}
& K_{0}^{*}=S_{3}\left(w_{0}, w_{1}, w_{2}, w_{3}\right) \\
& \quad \ldots \ldots \\
& K_{i}^{*}=S_{j}\left(w_{4 i}, w_{4 i+1}, w_{4 i+2}, w_{4 i+3}\right) \quad 3 \equiv(i+j) \bmod 8 \\
& \quad \ldots \ldots \\
& K_{65}^{*}=S_{2}\left(w_{260}, w_{261}, w_{262}, w_{263}\right)
\end{aligned}
$$

Step 4, let $K_{i}=K_{i}^{*} \oplus K_{i+33}^{*} \quad(0 \leq i \leq 32)$

Because the each byte Hamming weight of $K_{i}^{*}(0 \leq i \leq 65)$ cannot be gotten from the each byte Hamming weight of $K_{i}(0 \leq i \leq 32)$ the above attack cannot be carried out. But this schedule is slower than the original schedule. The second is as follows:

Step 1 and step 2 are same as the first schedule.

Step 3, let $v_{i}=w_{i} \oplus w_{i+132}(0 \leq i \leq 131)$.

Step 4, let $K_{i}=S_{j}\left(v_{4 i}, v_{4 i+1}, v_{4 i+2}, v_{4 i+3}\right), 3 \equiv(i+j) \bmod 8,(0 \leq i \leq 32)$.

The above attack can predict $v_{i}(0 \leq i \leq 131)$ but not $w_{0}, \ldots, w_{131}, \ldots, w_{263}$. So the second schedule is safe and its speed is nearly same as the original schedule.

\section{REFERENCES}

[1] P. Kocher, J.Jaffe, and B.Jun, "Differential Power Analysis", http://www.cryptography.com/dpa

[2] E. Biham, A. Shamir, "Power Analysis of the Key Scheduling of the 
AES Candidates", http://www.cs.technion.ac.il/ biham/

[3] R.L.Rivest, M.J.B.Robshaw, "RC6", http://www.nist.gov/aes.

[4] Ross Anderson, "SERPENT", http://www.nist.gov/aes. 